\title{
Dynamic Hysteresis in Finemet Thin Films
}

\author{
L. Santi, R. L. Sommer, A. Magni, G. Durin, F. Colaiori, and S. Zapperi
}

\begin{abstract}
We performed a series of dynamic hysteresis measurements on Finemet films with composition $\mathrm{Fe}_{73.5} \mathrm{Cu}_{1} \mathrm{Nb}_{3} \mathrm{Si}_{13.5} \mathrm{~B}_{9}$, using both the longitudinal magneto-optical Kerr effect (MOKE) and the inductive fluxometric method. The MOKE dynamic hysteresis loops show a more marked variability with the frequency than the inductive ones, while both measurements show a similar dependence on the square root of frequency. We analyze these results in the frame of a simple domain wall depinning model, which accounts for the general behavior of the data.
\end{abstract}

Index Terms-Domain wall dynamics, dynamic hysteresis, magnetic thin films, magnetization processes.

\section{INTRODUCTION}

$\mathbf{T}$ HE physics of thin and ultrathin magnetic films has been extensively studied in the recent past because of its great importance in several applications, ranging from multilayers to high-frequency devices. For this reason, many recent papers have been devoted to measure the magnetization reversal dynamics in two-dimensional (2-D) structures, revealing the existence of universal features and scale-invariant properties of the hysteresis loops [1]-[4]. Despite these efforts, a general description of these features is still an open problem, as most experimental results are still to be interpreted in the framework of the existing models [5]-[8]. In particular, the dynamic hysteresis loop area $A$ is often assumed to scale as $A \propto H_{0}^{\alpha} \omega^{\beta} T^{-\gamma}$, where $H_{0}$ is the amplitude of sinusoidal external field of frequency $\omega$, $T$ is the temperature, and $\alpha, \beta$, and $\gamma$ are three scaling exponents. As a matter of fact, experimental evaluation of the exponents $\alpha$ and $\beta$ in the low dynamic regime spans a quite large range from 0 to 0.8 , with a general higher value for thinner films [8], [9] (see also [10, Tab. I]). The proposed theoretical models roughly span the same range so that a clear identification of the fundamental properties of magnetization dynamics seems far to be reached. In order to investigate this complicated problem offering a new perspective, we present a series of dynamic hysteresis measurements on Finemet thin films by using the magneto-optical Kerr effect (MOKE), as employed in all the studies presented in the literature, and the fluxmetric inductive method, using a pick-up coil wound around the sample. This enables us to investigate the hysteresis properties not only considering the magnetization changes of the surface within the laser spot area

Manuscript received January 6, 2003.

L. Santi and R. L. Sommer are with the University Federal de Santa Maria, Department de Fisica UFSM, Santa Maria, RS, Brasil (e-mail: santi@ien.it; sommer@ccne.ufsm.br).

A. Magni and G. Durin are with the IEN Galileo Ferraris, 10135 Torino, Italy (e-mail: magni@ien.it, durin@ien.it).

F. Colaiori and S. Zapperi are with the INFM Unità di Roma 1, Dipartimento di Fisica, Università di Roma "La Sapienza," 00185 Roma, Italy (e-mail: fran@pil.phys.uniroma1.it; zapperi@pil.phys.uniroma1.it).

Digital Object Identifier 10.1109/TMAG.2003.815550 but those of the total volume of the sample as well; this is particularly important in order to check the dependence of the loop area on the film thickness and to understand the true nature of the magnetization dynamics. Quite unexpectedly, MOKE hysteresis loops show a remarkable variability with the frequency: about one order of magnitude higher than the inductive ones; on the other hand, both methods give a similar dependence on the frequency. We try to interpret these results with a simple domain wall depinning model that can be solved analytically, giving reasons for the general behavior of the data.

\section{Materials AND MEASUREMEnt Methods}

Films having nominal composition $\mathrm{Fe}_{73.5} \mathrm{Cu}_{1} \mathrm{Nb}_{3} \mathrm{Si}_{13.5} \mathrm{~B}_{9}$ have been deposited on glass substrates by $\mathrm{rf}$ magnetron sputtering under a 5 mTorr Ar atmosphere at room temperature. The sample thickness, ranging from about $21 \mathrm{~nm}$ to $5 \mu \mathrm{m}$, is measured by angle X-ray diffractometry, which also confirms the amorphous state of the samples. The hysteresis loops of the in-plane magnetization are measured in the as-prepared materials as a function of the applied field (up to a few kiliamps per meter) at $100 \mathrm{~Hz}$ and as a function of the frequency. The longitudinal MOKE measurements are performed with an optical bench equipped with an $\mathrm{He}-\mathrm{Ne}$ laser light source, covering a sample surface of about $1 \mathrm{~mm}^{2}$ and a photodiode having a frequency cutoff above $150 \mathrm{kHz}$. Samples are cut to a maximum size of about $2 \times 2 \mathrm{~mm}$ and inserted in a Helmholtz coil setup giving a maximum field of about $15 \mathrm{kA} / \mathrm{m}$. In this configuration, we could perform measurements up to $300 \mathrm{~Hz}$.

The fluxometric measurements of hysteresis loops are performed on larger samples, usually cut within an homogeneous region of 3 by $1.5 \mathrm{~cm}$. For these samples, we prepared a 10 -cm-long solenoid with 720 coils and an N/L value of 13200 . The sinusoidal applied field is measured detecting the voltage over a calibrated $1 \Omega$ resistance. The induced flux is detected with two sets of 50 coils wound around the sample and covering an area of about $1 \mathrm{~cm}$; the two sets are wired in order to cancel out the air flux and directly detect the film magnetization changes. As the film cross section is much smaller than the area of the coils, a perfect cancellation of the air flux is often difficult, requiring a continuous adjustment of area of one of the sets. This problem further increases at high frequencies, due to the different coupling between the wires, making it hard to perform the measurement. We thus get the full cancellation by numerically subtracting a sinusoidal wave with proper amplitude and phase from the induced signal. This procedure has a certain degree of arbitrariness, as small changes of the sinusoidal amplitude and phase give slightly different loop shapes, whereas the loop area and the coercive field are not substantially affected. 


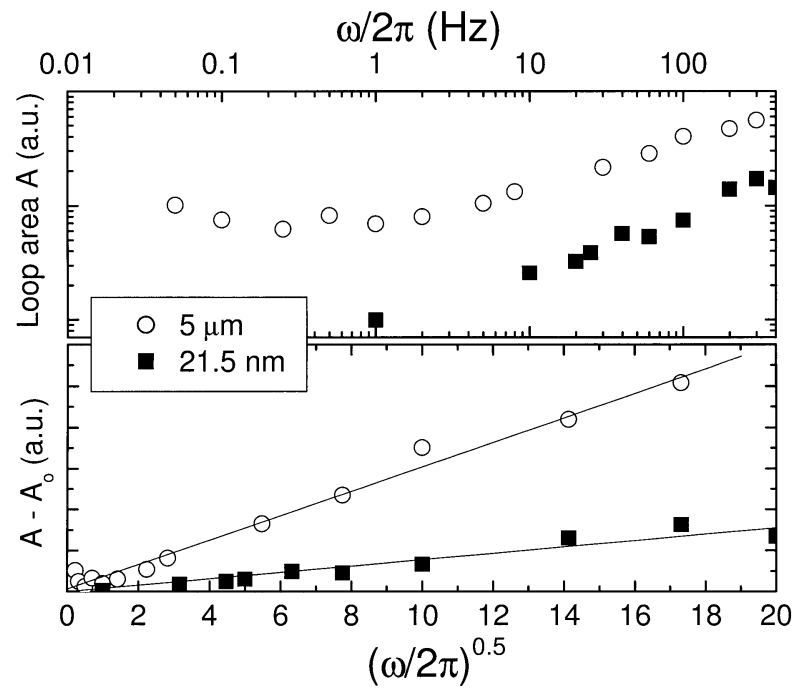

Fig. 1. MOKE measurements of the hysteresis loop area $A$ (top) for the thickest ( $5 \mu \mathrm{m}$-open dots) and the thinner (21 nm-squares) Finemet sample. Data for the $5 \mu \mathrm{m}$ would suggest the presence of a dynamic transition around a few Hertz. A plot of the area after subtraction of the static value $A_{0}$ (bottom) shows instead a linear dependence on the square root of frequency $(\beta=0.5$ in (1)).

\section{EXPERIMENTAL RESULTS}

The first fundamental result of both kind of measurements is the existence of a well-defined static hysteresis, as usually found in magnetic materials (see, for instance, [11]). Therefore, the loop area $A$ is better described by

$$
A \sim A_{0}+H_{0}^{\alpha} \omega^{\beta} T^{-\gamma}
$$

where the static loop area $A_{0}$ is estimated using data at the lowest frequencies. Clearly, the choice of (1) completely changes the experimental estimation of critical exponents. We believe that this simple observation could explain the so-called dynamic transition, which is a sharp change in the value of the exponent $\beta$ at intermediate frequencies. Due to the usual large value of $A_{0}$ with respect to variation of the data with the frequency, log-log plots can mimic a dynamic transition when data actually follow a simple power law, as assumed in (1). Our results and the theoretical analysis should help to clarify this important point.

The MOKE hysteresis loop area for different sample thickness show large variations with the frequency, as shown in Fig. 1 for the thickest $(5 \mu \mathrm{m})$ and thinnest $(21 \mathrm{~nm})$ samples. Visual inspection of Fig. 1 would suggest a dynamic transition around a few Hertz, at least, for the 5- $\mu$ m sample. As a matter of fact, the data show a much simpler behavior; in fact, the loop area $A$ follows a simple law of the type $A \simeq A_{0}+k \omega^{0.5}$, where $A_{0}$ is estimated using low-frequency data.

Surprisingly, hysteresis loops obtained with the fluxometric setup do not show the same frequency variability. As reported in Fig. 2, the loop area changes only about $10 \%$ with respect to the static value $A_{0}$, even if we consider a much larger frequency range (up to $2 \mathrm{kHz}$ ) in comparison with MOKE measurements. On the other hand, when plotted as a function of the square root of frequency, these data follow a linear behavior reasonably
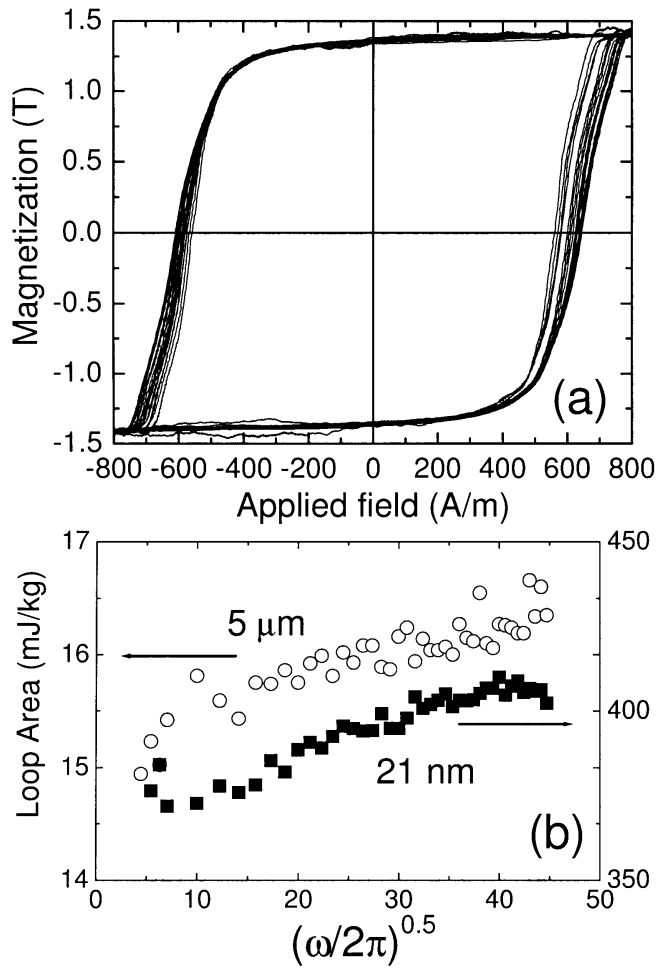

Fig. 2. (a) Hysteresis loops of a 21-nm $\mathrm{Fe}_{73.5} \mathrm{Cu}_{1} \mathrm{Nb}_{3} \mathrm{Si}_{13.5} \mathrm{~B}_{9}$ Finemet film up to $2 \mathrm{kHz}$, with an external field amplitude of $800 \mathrm{~A} / \mathrm{m}$. (b) Frequency dependence of loop area of the same sample and of a $5-\mu \mathrm{m}$ film. Note the approximately linear dependence as a function of the square root of the frequency.

well. This suggests a common mechanism responsible for the magnetization change, which we try to interpret considering the domain wall depinning models, as discussed below.

Inductive measurements of the hysteresis loops at $100 \mathrm{~Hz}$ as a function of the applied field amplitude do not show any scaling behavior as given by (1) or similar. Simple "squared loops" such as the ones shown in Fig. 2 are observed only for the thinner films, whereas more complicated shapes appear for thicker samples, showing a clear evidence of multidomains magnetization processes. We postpone the discussion of these rich but complex features to a further longer publication.

\section{MODEL}

To understand the frequency dependence of our experimental data, we employ the domain wall depinning model described in [1]. Under the assumption that hysteresis is mainly due to domain wall motion, we consider a phenomenological law for the wall velocity given by

$$
v(H)=\mu\left(|H|-H_{p}\right) \theta\left(|H|-H_{p}\right)
$$

where $\mu$ is the wall mobility, $H_{p}$ is the depinning field, $\theta$ is the step function, and the applied field is $H=H_{0} \sin (\omega t)$. Following [6], we solve (2) and compute the coercive field and the loop area, which at low frequency are given by

$$
H_{c}(\omega) \simeq H_{p}+\left(2 L \omega \sqrt{\frac{H_{0}^{2}-H_{p}^{2}}{\mu}}\right)^{1 / 2}
$$




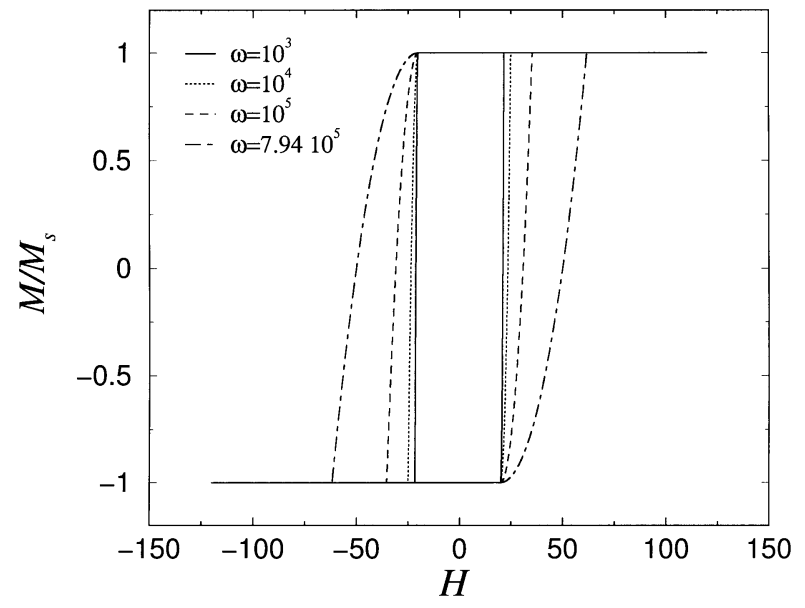

Fig. 3. Dynamic hysteresis loops calculated using the simple domain wall model of (2). Hysteresis branches are described by (4).

where $L$ is the sample size, in the case of a single domain wall, or the typical distance between domain walls in a more general case. The lower branch of the hysteretic loop is then given by

$$
M(h)= \begin{cases}-M_{s} & H<H_{p} \\ M_{s}\left(\frac{\mu}{L \omega} \frac{\left(H-H_{p}\right)^{2}}{\sqrt{H_{0}^{2}-H_{p}^{2}}}-1\right), & H \geq H_{p}\end{cases}
$$

where the second equation is valid as long as $M<M_{s}$. Fig. 3 displays the loop shapes for different values of frequency $\omega$. The loop area is thus easily computed and, for low frequencies, is given by

$$
A \simeq A_{0}+M_{s} \frac{8}{3} \sqrt{\frac{2 \omega L}{\mu} \sqrt{H_{0}^{2}-H_{p}^{2}}}
$$

where $A_{0}=4 M_{s} H_{p}$ is the area of the loop in the quasistatic limit.

\section{Discussion AND CONCLUSION}

The simple model shown above gives a simple $\sqrt{\omega}$ dependence of the loop area (5), which we believe can account for some experimental data found in the literature and interpreted assuming the presence of a dynamic transition. The value $\beta=$ 0.5 is a strict consequence of the linear form of (2); in a more general case, shown in detail in [6], one gets $\beta \geq 0.5$. It is worth noting that the model above describes the magnetization dynamics of a single domain wall having a well-defined depinning field $H_{p}$. The model does not include the effects of any random disorder, a multiplicity of domains, or a field-dependent nucleation of domain walls. As a consequence, it cannot describe minor hysteresis loops or other features, such as more complicated shapes of the loops. Despite these limitations, we believe it can account for the general behavior of the dynamic hysteresis and could be successfully applied to describe thin films showing simple magnetization dynamics.

Using the results of the model, we found that our experimental data obtained with complementary measurement techniques are the consequence of the same magnetization dynamics. That means that the MOKE measurements on the surface are related to those of the volume, which are given by the inductive method. While this is expected in thin films, it is not necessarily valid for the thickest samples, for instance, in the case of a few microns. It is not in any way clear what can account for the large differences in the change of the loop area. It is worth noting that the MOKE measurement uses a laser spot area close to the sample dimension; therefore, in principle, it should detect the magnetization changes of the entire sample, as long as we consider the thinnest films. Therefore, we should observe similar variations using the two methods. On the other hand, one can suggest that samples with the same thickness but different lateral dimensions could not have the same magnetization dynamics because of the different role of the demagnetizing fields or of the number of domain walls. This possibility has been ruled out by performing fluxometric measurements on the same sample used in MOKE, having thickness $5 \mu \mathrm{m}$. In this case, the fluxometric measurements show the same behavior described in Fig. 2. Unfortunately, measurements at lower thickness are not possible due to the very low intensity of the induced signal, given the reduced cross section available. However, we feel confident that this result proves that the sample lateral dimensions are not relevant, also considering that the data reported in literature refer to variations only on the sample thickness and not, more generally, on the other two dimensions.

In summary, we have shown in this paper that the dynamic hysteresis of $\mathrm{Fe}_{73.5} \mathrm{Cu}_{1} \mathrm{Nb}_{3} \mathrm{Si}_{13.5} \mathrm{~B}_{9}$ films exhibit different behavior as a function of sample thickness and magnetizing field frequency and that the domain wall depinning model accounts for the general behavior of the data.

\section{REFERENCES}

[1] I. Ruiz-Feal, T. A. Moore, L. Lopez-Diaz, and J. A. C. Bland, "Model for reversal dynamics of ultrathin ferromagnetic films," Phys. Rev. B, vol. 65, p. $054409,2002$.

[2] B. Choi, W. Y. Lee, A. Samand, and J. A. C. Bland, "Dynamics of magnetization reversal in thin polycrystalline $\mathrm{Ni}_{80} \mathrm{Fe}_{20}$ films," Phys. Rev. B, vol. 60, pp. 11 906-11909, 1999.

[3] Q. Jiang, H.-N. Yang, and G.-C. Wang, "Scaling and dynamics of lowfrequency hysteresis loops in ultrathin co films on a $\mathrm{Cu}(001)$ surface," Phys. Rev. B, vol. 52, pp. 14911-14916, 1995.

[4] Y.-L. He and G.-C. Wang, "Observation of dynamic scaling of magnetic hysteresis in ultrathin ferromagnetic $\mathrm{Fe} / \mathrm{Au}(001)$ films," Phys. Rev. Lett., vol. 70, pp. 2336-2339, 1993.

[5] F. Zhong, "Monte carlo renormalization group study of the dynamic scaling of hysteresis in the two-dimensional ising model," Phys. Rev. $B$, vol. 66, p. $060401,2002$.

[6] I. F. Lyuksyutov, T. Nattermann, and V. Pokrovsky, "Theory of the hysteresis loop in ferromagnets," Phys. Rev. B, vol. 59, pp. 4260-4272, 1999.

[7] B. K. Chakrabarti and M. Acharyya, "Dynamic transition and hysteresis," Rev. Mod. Phys., vol. 71, pp. 847-859, 1999.

[8] W. Y. Lee, B.-C. Choi, Y. B. Xu, and J. A. C. Bland, "Magnetization reversal dynamics in epitaxial Fe/GaAs(001) thin films," Phys. Rev. B, vol. 60, pp. 10216-10221, 1999.

[9] T. A. Moore, J. Rothman, Y. B. Xu, and J. A. C. Bland, "Thickness-dependent dynamic hysteresis scaling behavior in epitaxial $\mathrm{Fe} / \mathrm{GaAs}(001)$ and $\mathrm{Fe} / \mathrm{InAs}(001)$ ultrathin films," J. Appl. Phys., vol. 89, pp. 7018-7020, 2001.

[10] J.-S. Suen and J. L. Erskine, "Magnetic hysteresis dynamics: Thin p1 $\times 1$ ) Fe films on flat and stepped W(110)," Phys. Rev. Lett., vol. 78, pp. 3567-3570, 1997.

[11] G. Bertotti, Hysteresis in Magnetism. San Diego, CA: Academic, 1998. 